\title{
Patterns and Causes of Amputation in Ayder Referral Hospital, Mekelle, Ethiopia: A Three-Year Experience
}

\author{
Berhe Gebreslassie $^{1^{*}}$, Kibrom Gebreselassie ${ }^{2}$, Reiye Esayas ${ }^{2}$
}

\section{OPEN ACCESS}

Citation: Berhe Gebreslassie, Kibrom Gebreselassie, Reiye Esayas. Patterns and Causes of Amputation in Ayder Referral Hospital, Mekelle, Ethiopia: A ThreeYear Experience. Ethiop J Health Sci.2017;28(1):31

doi:http://dx.doi.org/10.4314/ejhs.v28i1.5

Received: July 31, 2017

Accepted: August 1, 2017

Published: January 1, 2018

Copyright: (c) 2018 Berhe Gebreslassie.,

et al . This is an open access article distributed under the terms of the

Creative Commons Attribution License,

which permits unrestricted use,

distribution, and reproduction in any

medium, provided the original author and source are credited.

Funding: Nil

Competing Interests: The authors declare that this manuscript was approved by all authors in its form and that no competing interest exists.

Affiliation and Correspondence:

${ }^{1}$ Orthopedics Unit, College of Health

Sciences, Mekelle University

${ }^{2}$ Department of Surgery, College of

Health Sciences, Mekelle University

*Email:berhe5907@yahoo.com

\begin{abstract}
BACKGROUND: Amputation is a surgical procedure for the removal of a limb which is indicated when limb recovery is impossible. There are different types of amputation, and their causes can vary from one area to the other. Therefor, the aim of this study is to find out the patterns and causes of amputations in patients presented to Ayder Referral Hospital, Mekelle, Ethiopia.

METHODS: the record of 87 patients who had amputation at different sites after admission to Ayder referral hospital, Mekelle, Ethiopia in three years period were reviewed retrospectively. RESULT: A total of 87 patients had amputation of which $78.2 \%$ were males. The age range was from 3 to 95 years, and the mean age was 40.6 in years. The most common indications were trauma (37.7\%), tumor (24.1\%), and peripheral arterial disease (PAD) (20.7\%). The commonest type of amputation was major lower limb amputation (58.6\%) which includes above knee amputation (35.6\%)and below knee amputation (23\%) followed by digital amputation (17.2\%). There was 11.4\% major upper limb amputation of which there was one patient who had re-amputation. CONCLUSION: Most of the indications for amputations in our setup are potentially preventable by increasing awareness in the society on safety measures both at home and at work and early presentation to health facilities.
\end{abstract}

KEYWORDS: Amputation. Amputation pattern, amputation indications

\section{INTRODUCTION}

Amputation is a surgical procedure for the removal of a limb which is indicated when limb recovery is impossible or when the limb is dead or nonfunctional risking the patient's life. It is one of the oldest surgical procedures with a history of over 2500 years going back to the time of Hippocrates (1). The leading causes of limb amputation varies from one country to the other; there is also variation from one city to the other (2). In developed countries, peripheral vascular disease or complications related to Diabetes mellitus ranks first as a cause for amputation whereas trauma, infections, uncontrolled diabetes mellitus and malignancies are still the leading causes for amputation in developing countries $(3,4)$. 
As far as the site of amputation is concerned, studies showed different results, but lower-limb amputations account for $79 \%$ of all dysvascular limb loss discharges from hospital (5). The pattern of limb amputation in a low income country also showed, in descending order, trans-tibial, transfemoral, trans-radial, and trans-humeral (6). In Ethiopia, vascular disease, apart from diabetes, is less common and amputation is often the only available treatment for the late results of trauma; mainly, for late complications, for gangrene of various causes, for tumors and for chronic infections (7). The majority of these amputations are preventable by empowerment of traffic rules, health education, early preventions and appropriate management of the common infections (6). The objective of this study is to know the pattern and causes of amputation in Ayder Referral Hospital, Mekelle. It can be used as a bridge to undertake further researches on the types and indications of amputation in the region and in the country as a whole, so that we can at least protect our society from having these disabling problem by creating awareness on causes which can be preventable.

\section{PATIENT AND METHODS}

This was a cross-sectional retrospective review of records from January 1, 2011 to December 31, 2013, performed at Ayder Referral Hospital, Mekelle, Ethiopia. This hospital has one orthopaedic surgeon who receives referral from within and outside of Mekelle. Ethical approval for use of patient records was obtained from the hospital medical director and ethical committee of the College of Health Sciences, Mekelle University. Data were reviewed from surgical and orthopedics $\log$ books found in the operation theater. From the log books, all patients who had undergone amputation were selected and their charts were retrieved based on the medical recording number. Incomplete or lost charts were excluded. A pretested questionaire was used to collect data by trained general practitioners. Data were entered by the general practitioners and cross checked by the supervisors, prior to the beginning of analysis. Accuracy of data was verified by entering data twice for analysis on SPSS window version 16.0.

\section{RESULT}

A total of 87 patients underwent limb amputations during the study period, of which 68 were males $(78.2 \%)$ with a male to female ratio of 3.6:1. The mean age in years was 40.6 and the range was from 3 to 95 (Table 1). The causes of amputation are shown in Table 2 . In fact, $13.8 \%$ of patients had a treatment by traditional healers before they came to our hospital.

Table 1: Age distribution of patients who underwent amputation in ARH from January 1, 2011 to December 31, 2013.

\begin{tabular}{lll}
\hline Age in years & Number of patients & $\%$ \\
\hline $0-15$ & 13 & 14.9 \\
$16-30$ & 24 & 27.6 \\
$31-45$ & 13 & 14.9 \\
$46-60$ & 18 & 20.7 \\
$61-75$ & 12 & 13.8 \\
$>75$ & 7 & 8 \\
\hline
\end{tabular}

Table 2: Causes of amputation in Ayder referral hospital.

\begin{tabular}{lll}
\hline Causes of Amputation & Number & $\mathbf{\%}$ \\
\hline Tumor & 21 & 24.1 \\
Peripheral arterial disease(PAD) & 18 & 20.7 \\
Trauma & & \\
$\quad$ Fall down accident (FDA) & 9 & 10.3 \\
Electrical burn & 9 & 10.3 \\
Machine injury & 5 & 5.7 \\
$\quad$ Road trafic accident (RTA) & 5 & 5.7 \\
$\quad$ Others(bullet, other burns) & 5 & 5.7 \\
Infection & 8 & 9.2 \\
Diabetic foot ulcer & 7 & 8 \\
\hline
\end{tabular}


Patterns and Causes of Amputation...

Berhe G. et al.

In patients under the age of 15 , trauma specifically FDA was the commonest cause of amputation while, in the age group between 15 to 45 malignancy and electrical burn were the commonest causes. After the age of 45, malignancy and PAD were the commonest causes (Table 3).

The commonest type of amputation was major lower limb amputation (58.6\%) which included
AKA (35.6\%) and BKA (23\%) followed by digital amputation (17.2\%). Major upper limb amputation was $11.4 \%$ of which there was one patient with bilateral above elbow amputation. The proportion of major limb amputation showed a ratio of 1.55:1 for AKA to BKA in the lower limb and 3.5:1 ratio for AEA to BEA in the upper extremity.There was also one patient who had bilateral above elbow amputation. (Figure 1).

Table 3: Distribution of causes of amputation among different age groups in ARH from January 1, 2011 to December 31, 2013

\begin{tabular}{lcccccccccc}
\hline $\begin{array}{l}\text { Age } \\
\text { group }\end{array}$ & Tumor & PAD & FDA $\begin{array}{c}\text { Electric } \\
\text { Burn }\end{array}$ & $\begin{array}{c}\text { RTA } \\
\text { Muchine }\end{array}$ & $\begin{array}{c}\text { Mathers } \\
\text { injury }\end{array}$ & Infection DFU & Total \\
\hline $0-15$ & 1 & 0 & 6 & 2 & 0 & 0 & 0 & 1 & 0 & 13 \\
$16-30$ & 7 & 0 & 4 & 6 & 1 & 2 & 3 & 3 & 0 & 24 \\
$31-45$ & 1 & 3 & 0 & 1 & 1 & 1 & 1 & 2 & 3 & 13 \\
$46-60$ & 5 & 6 & 0 & 0 & 1 & 2 & 1 & 1 & 3 & 18 \\
$61-75$ & 6 & 3 & 1 & 0 & 0 & 0 & 0 & 1 & 1 & 12 \\
$>75$ & 1 & 6 & 0 & 0 & 0 & 0 & 0 & 0 & 0 & 7 \\
Total & 21 & 18 & 11 & 9 & 3 & 5 & 5 & 8 & 7 & 87 \\
& & & & & & & & & & \\
\hline
\end{tabular}

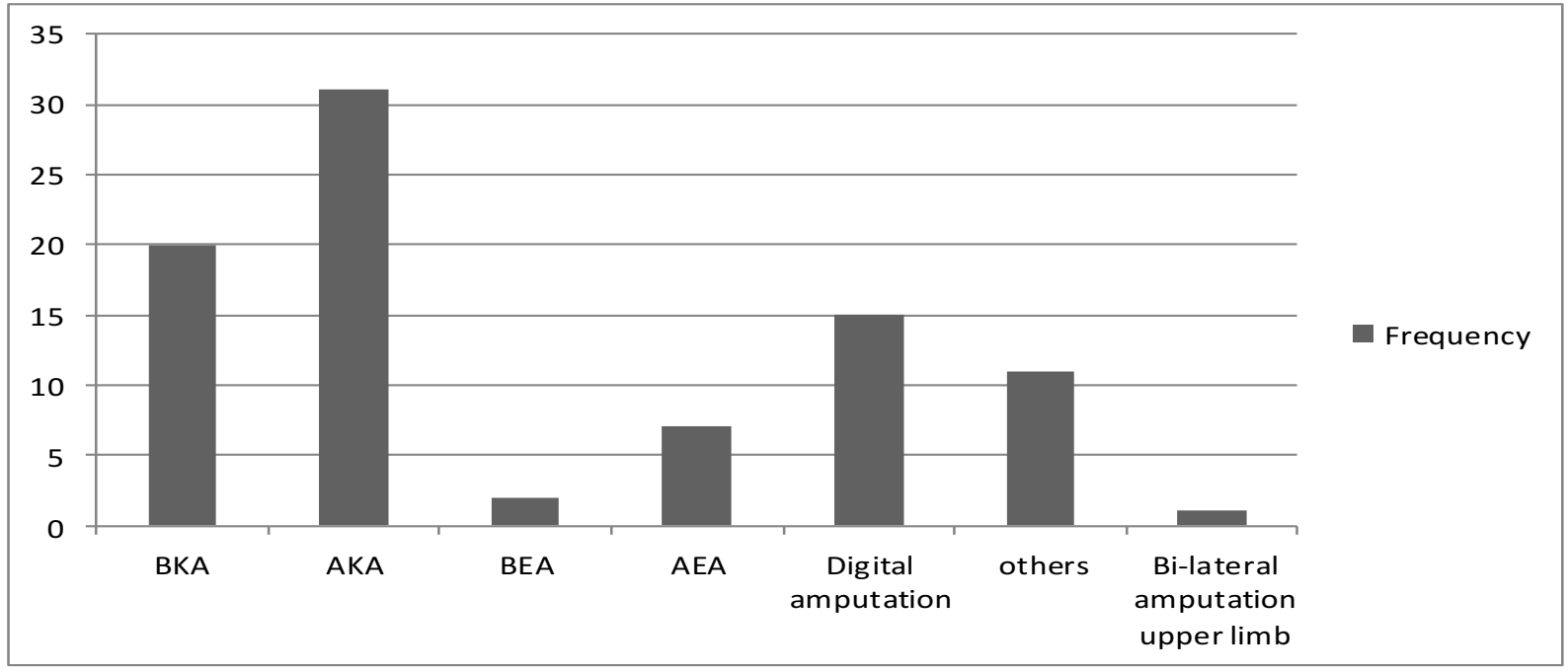

Type of amputation done

Figure 1: The types of amputation in Ayder referral hospital from January 1, 2011 to December 31, 2013

DOI: http://dx.doi.org/10.4314/ejhs.v28i1.5 


\section{DISCUSSION}

Amputation is the most ancient of all surgical procedures. It was first described by Hippocrates in 460-377 BC; since then, it is used as one treatment modality by orthopedics, general, trauma, and vascular surgeons to save the life of many patients (8). The causes and pattern of limb amputation vary from one hospital to the other in a country. There is also variation from one country to the other. So this research was done to see the pattern and causes of amputation in Ayder Referral Hospital and also compares it with other similarly studies. This will help to identify the preventable condition and recommend to the responsible stakeholders.

In this study, 68 of the patients were males (78.2\%). Similar results were also reported from Back Lion Hospital in Ethiopia (76\%), Tanzania (66.7\%), Sudan (76\%) and Iran (79.62\%) $(9,10,11)$. It can be explained by our culture that males are involved in different activities that can expose them to traumas. As to the age, it is more common in age groups of $16-30$ and $46-60$ years which is similar to the studies done in Tanzania and Sudan $(9,10)$, but in contrast to ours, a study done in Ghana showed high incidence in the 7th decade (12). This can be explained by the different indications and pattern of the amputation.

Even though there is variation in the causes of amputation in different age groups, trauma (includes RTA, burn, FDA, others) $(37.7 \%)$ and tumor $(24.1 \%)$ were the two common causes in our setup. There were also similar results from studies in Kenya $(26.5 \%$ for both causes) and Nigeria (34\% for trauma and $14.5 \%$ for tumor) $(13,14)$. This indicates that our safety rules for trauma are very poor, and regarding tumor, late presentation of patients and absence of limb sparing surgery facility in our setup are the main explanations.

Trauma accounted for 33 patients $(37.7 \%)$ of all causes of amputation in our study. The major causes of trauma were FDA which was seen in 11 patients of which 6 were under 15year of age and 5 were between the ages of 16-30 years followed by an electrical burn in 9 patients, of which 6 were between 16-30 years. The other traumas were machine injury in 5 patients, RTA in 3 patients, and 5 others which includes bullet injury, inappropriate splintage by traditional bone setters and other burn injuries. The majority of these cases were preventable by involving different levels and parts of professionals to give health education, on work and traffic safety rules to different workers and children to create awareness to the society at all possible occasions. The reports from Tanzania and Kenya also showed burn and FDA as major causes of amputation even though RTA outnumbers $(9,15)$.

Tumor, as an indication of amputation, showed a bimodal distribution over age groups of 16-30 and 46-60 years. PAD was the third cause of amputation in our study, and it was mainly seen after the age of 45 years in 15 patients out of 18 . In the developed world, PAD is diagnosed, and the extent of the disease is decided by investigation like Ankle-brachial index, Duplex ultrasound, Angiography, Computed tomography angiography, and Magnetic resonance angiography in addition to different laboratory tests (16). In our opinion, absence of those especial investigations were the contributing factors for the delay of diagnosis and early intervention in our hospital. A similar study done in Tanzania on 244 patients showed that tumor and PAD were the commonest causes of amputation (17). PAD is also the commonest cause of amputation in the developed countries even in the presence of all the necessary investigation $(18,19,20)$.

In several studies, diabetic foot ulcer was the commonest indication of amputation $(21,22)$, but this is not in agreement with our study which showed that DFU is the $5^{\text {th }}$ cause next to severe infection which is not possible to eradicate with surgery and antibiotic. We did not get any indication of congenital defects as a cause of amputation, like the majority study done in other places.

Looking at the sites of amputation, major lower limb amputations were more common than

DOI: http://dx.doi.org/10.4314/ejhs.v28i1.5 
upper limb amputations which is similar to other studies done in Tanzania, Sudan and Nigeria. Among those lower limb amputations the most frequent site of amputation in our setup was above knee amputation but the study from Tanzania, and Nigeria showed that below knee amputation was more common $(4,9,10,14)$. In our personal experience, the important reason is that most patients with tumor and peripheral arterial disease conditions had above knee amputation because we did not have the necessary investigation to localize the extension of the disease.

Trauma and tumors were the common indications for amputations in our setup. Most of these conditions are potentially preventable by early presentation and proper treatment. Trauma causes can be prevented by increasing awareness in the society to have both home and work safety measures. It is also possible to decrease the risk by bringing patients early to a health facility. The awareness of tumor and early visit should be done in addition to the strengthening of the health services like limb sparing surgery.

This study should be extended to regional and national levels, so that we can have more data to identify the causes and levels of amputations.

\section{REFERENCES}

1. Paudel B, Shrestha BK, Banskota AK. Two faces of major lower limb amputations. Kathmandu Univ Med $J$ (KUMJ). 2005;3(3):212-216.

2. Thanni L. O., Tade A. O. Extremity amputation in Nigeria - a review of indications and mortality. Surgeon. 2007; 5(4): 213-217.

3. Abou-Zamzam AM, Jr, Teruya TH, Killeen JD, Ballard JL. Major lower extremity amputation in an academic vascular center. AnnnVasc Surg. 2003;17(1):86-90.

4. Olasinde AA, Oginni LM, Bankole JO, Adegbehingbe, Oluwadiya KS. Indications for amputations in Ile-Ife, Nigeria. Niger $J$ Med. 2002;11(3):118-121.

5. National Limb Loss Information Centre Fact Sheet, Amputation Statistics by Cause Limb
Loss in the United States, Revised 2008 (reviewed on October 14, 2011).

6. Soomro N, Bibi R, Ahmed SI, Kamran B, Minhas MA, Siddiqui KY. Epidemiology of amputation; low resource community: Sindh Province, Pakistan. Professional Med J 2013;20(2): 261-265.

7. An Introduction To Orthopaedics, Department of Orthpaedic Surgery, AAUMedical Faculty, 1994; Pp 1 -77.

8. Van der Meij W: K N: No leg to stand on. Historical relation between amputations. Surgery and Prostheseology 1995, 1:1-256.

9. Chalya PL, Mabula JB, Dass RM, Ngayomela IH, Chandika AB, Mbelenge N, et al. Major limb amputations: A tertiary hospital experience in northwestern Tanzania. Journal of Orthopaedic Surgery and Research 2012; 7:18

10. Doumi EA, Ali AJ. Major limb amputations in El Obeid Hospital, Western Sudan. Sudan JMS. 2007; 2(4): 237-239.

11. Sarvestani AS, Azam AT. Amputation: A Ten-Year Survey. Trauma Mon. 2013 December; 18(3): 126-129.

12. Naaeder SB: Amputation of the lower limb in Korle-Bu Teaching hospital, Accra. West Afr J Med 1993;12:21-26.

13. Muyembe VM, Muhinga MN. Major limb amputation at a provincial general hospital in Kenya. East Afr Med J 1999;76:163-166

14. Thanni LO, Tade AO. Extremity amputation in Nigeria-a review of indications and mortality. Surgeon 2007;5:213-217.

15. Ogeng'o JA, Obimbo MM, King'ori J. Pattern of limb amputation in a Kenyan rural hospital. International Orthopaedics (SICOT) 2009; 33:1449-1453.

16. European Society of Cardiology Guidelines on the diagnosis and treatment of peripheral artery diseases. 2011. Pp 8-9.

17. Loro A, Franceschi F. Prevalence and causal conditions for amputation surgery in the third world: ten years experience at Dodoma Regional Hospital, Tanzania. Prosth Orth Int 1999;23:217-224.

18. Naraysingh $\mathrm{V}$, Singh $\mathrm{M}$, Raindass $\mathrm{Mj}$, Rampaul R, Ali T, Teeluck Singh S, Muharaj

DOI: http://dx.doi.org/10.4314/ejhs.v28i1.5 
D Major lower limb amputations in Trinidad; a retrospective analysis. (Amputations). Diabetic Foot. 2002, http://www.articlearchives.com/medicinehealth/diseases-isordersendocrine/9787791.html.

19. Polyolainen J, Alaranta H. Lower limb amputations in Southern Finland 1984-85. Prosth Orth Int 1988;12(1):9-18.

20. Rommers GM, Vos LD, Groothoff JN, Schuiling CH, Eisma WH. Epidemiology of lower limb amputees in the North of Netherlands, aetiology, discharge destination and prosthetic use. Prosth Orth Int 1997;21(2):92-99.

21. Trautner C, Haastert B, Spraul M, Giani G, Berger M. Unchanged incidence of lower limb amputations in a German city, 19901998. Diabetes Care 2001;24:855.

22. Dangelser G, Besson S, Gatina J, Blickle I. Amputations among diabetes in Reunion Island. Diabetes Metab 2003;29:628-34.

DOI: http://dx.doi.org/10.4314/ejhs.v28i1.5 